\title{
Stability Analysis and Optimization of Airport Security System
}

\author{
Yang $\mathrm{Li}^{1,3}$, Lanya $\mathrm{Ma}^{2,3}$, Yuanbiao Zhang ${ }^{3}$ \& Weijie Yang ${ }^{3,4}$ \\ ${ }^{1}$ Business Administration Department of International Business School, Jinan University, Zhuhai, China \\ ${ }^{2}$ Finance Department of International Business School, Jinan University, Zhuhai, China \\ ${ }^{3}$ Innovation Practice Base of Mathematical Modeling, Jinan University, Zhuhai, China \\ ${ }^{4}$ International Business Department of International Business School, Jinan University, Zhuhai, China \\ Correspondence: Yang Li, Business Administration Department of International Business School, Jinan \\ University, Qianshan Road 206\#, Zhuhai City, Guangdong Province, Post No. 519070, China. E-mail: \\ 15521319079@163.com
}

Received: February 24, 2017

Accepted: March 6, 2017

Online Published: March 20, 2017

doi:10.5539/emr.v6n1p56

URL: http://doi.org/10.5539/emr.v6n1p56

\begin{abstract}
In this paper, we have established a composite queuing network model on the basis of the queuing theory and combining with the current security system of the airport. We have made division of the whole security system into different processes which include ID check process, x-ray scanning process, and millimeter scanning process, and find out the unit of security system according to the proportion of different security lanes, what's more, these processes were distinguished into different kinds of queuing system, and then, we established the queuing network using these different queuing model into the unit. We applied the method of computer simulation to analyze the bottleneck, and we find that ID check process in pre-check system is unstable based on the current proportion of pre-check lane. Meanwhile, we optimized the number of each unit using the system design model and prove the effectiveness by computer simulation, then, we optimized the service rate of the baggage and body screening in pre-check lane follow the above process. Finally, we further discussed development direction of this model.
\end{abstract}

Keywords: composite queuing network model, system stability, computer simulation, System Design Optimization

\section{Introduction}

During 2016, U.S. Transportation Security Agency (TSA) came under sharp criticism for extremely lengthy lines, in particular at Chicago's O'Hare international airport. There have also been incidents of unexplained and unpredicted long lines at other airports, including airports that normally have short wait times. The high variance in checkpoint lines can be extremely costly to passengers as they decide between arriving unnecessarily early or potentially missing their scheduled flight. As a result, the airport security system optimization issues aroused the concern of all sectors of society.

Existing research on airport queuing system, which mainly uses the methods of integer programming, queuing theory, Logit model, and Petri network combining with service model, Pro model and other simulation software, mainly focus on check-in and security system. Yan, Nico M. van Dijk, and others used the method of integer programming to study the number of check-in counters, and get the ideal results (Yan, Tang, \& Chen, 2004; Van Dijk \& Van Der Sluis, 2006). Wai and Soemon Takakuwa applied the method of simulation to the international departure passenger flow in an airport terminal, and achieve significant results (Chun \& Raymond, 1999; Takakuwa \& Oyama, 2003). Gkritza Kestablishedpolynomial Logit model to analyze the security and service time of the security system, and find that service time is the decisive factor of passenger's satisfaction (Gkritza, Niemeier, \& Mannering, 2006). Guanghui Gu and Jingli Liufrom Nanjing University of Aeronautics and Astronautics analyzed the terminal departure process using Service model and Pro mode software respectively, finally, a complete simulation system of departure process was established with the idea of block modeling (Gu, $\mathrm{Xu}, \& \mathrm{Xia}, 2011)$. Naiyi Li studied the dynamics of departure flights with service model software, and improve the operational efficiency of the airport $(\mathrm{Li}, 2012)$. Regattieri from the University of Bologna applied the queuing theory to studying the amount of security door, and establish the M/M/M model (Regattieri, Gamberini, Lolli, \& Manzini, 2009). Yuehui Yang, Jinhua Gao and others from the Civil Aviation University of China using 
the queuing theory to analyze the capacity of each part of the passenger departure process, and the threshold for the capacity was found (Yang \& Gao, 2005). Jaromír Širokýfrom the University of Pardubice took the process of check-in and security services as the research object to establish the queuing theory model, and optimized the air transportation level and service level index using the Tecnomatix simulating software (Široký \& Hlavsová, 2014). Yuanyuan Guo and her partner focus on the passenger volume combining with a variety of mathematical analysis methods which contains chaos theory, wavelet denoising method, and RBF neural network chaotic time series, and short-term passenger terminal traffic flow forecasting model is established (Guo, 2013). Shaowu Cheng, Yaping Zhang and others from School of Computer Science and Technology in Harbin Institute of Technology analyzed the dynamic allocation and optimization of terminal resources using neural network, wavelet denoising method, and the effectiveness is proved by simulating (Cheng, Zhang, \& Bie, 2015).

This paper focuses on the stability of the current security system in the airport, analyzing the bottleneck of its processes, and optimizes the allocation of system resources based on queuing theory.

\section{Preparation}

Airport security program is composed of three systems which contain ID check, baggage and body screening and collect items and exist, and the main systems are ID check and baggage and body screening which can be seen as a different queuing system. The security system contains two different kinds of programs which are general security system and pre-check system. Approximately $45 \%$ of passengers enroll in a program called Pre-Check for trusted travelers. Pre-Check passengers are not required to remove shoes, belts, or light jackets, and they also do not need to remove their computers from their bags so that they can reduce their retention time in the baggage and body screening system.

The queuing system consists of three processes: the input process, the queuing rules, and the service mechanism.

\subsection{Input Process}

The input process mainly describes the source of the passengers and what regular the flow of passengers obeys when reaching the system.

(1) The quantity of passengers: For the airport, as long as the airport itself works well, passengers will continue to arrive. Therefore, passengers can be considered to be infinite.

(2) The reach of passengers: In general, passengers take specific flights, so passengers will arrive in batches.

(3) The distribution of the time intervals that passengers arrive one after another: According to the queuing theory, there are some common kinds of distribution: fixed-length distribution $\mathrm{D}$, negative exponential distribution M, Erlang distribution $\mathbb{E}_{\mathbb{R}}$, arbitrary distribution $\mathrm{G}$ (Han, 2005). Figure 1 shows the distribution of the time intervals of the passengers reaching the system through statistical analysis based on the data of 2017 ICM problem D. It reveals that the time interval of the passengers reaching the system roughly follows a negative exponential distribution. Therefore, we assume that the passenger arrival time interval obeys a negative exponential distribution and prove the correctness of the hypothesis through the Kolmogorov-Smirnov test. The results of the verification are in Table 1.
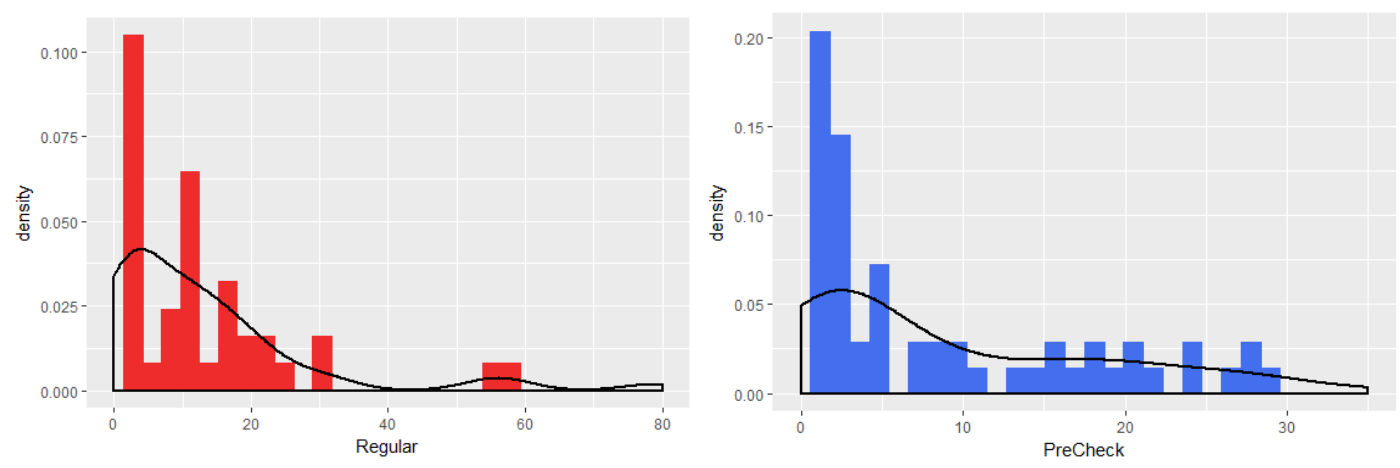

Figure 1. The arrival times interval distribution of TSA pre-check and regular pay passengers 
Table 1. The Kolmogorov-Smirnov test of single sample

\begin{tabular}{lccc}
\hline Check items & Attribute & Regular Pay & TSA Pre-Check \\
\hline $\mathrm{N}$ & & 57 & 45 \\
Exponential parameter & Mean & 9.1895 & 13.1556 \\
& Absolute & 0.181 & 0.17 \\
Most extreme differences & Positive & 0.181 & 0.17 \\
& Negative & -0.111 & -0.073 \\
Kolmogorov-Smirnov Z & & 1.165 & 1.141 \\
Asymp. Sig. (2-tailed) & & 0.065 & 0.148 \\
\hline
\end{tabular}

Table 1 shows that the data obeys exponential distribution which is according to the statistics of passengers' arrival time interval. According to the inspection results, $0.0650 .148>0.5$, the assumption that the passengers' arrival time interval obeys the negative exponential distribution with the acceptance degree of $95 \%$.

\subsection{Rule of Queuing}

(1) Capacity of queues: we can divide queues into two kinds: limited capacity and infinite capacity, and airport security system capacity limited.

(2) Rule of queuing: queuing rules include mainly FCFS (first come first service), LCFS (last come first service), PS (Priority service). The queuing rule of FCFS is most common in our daily life. Airport security system also obeys this rule.

(3) Service mechanism: the number of services stations is determined by the real situation, the form of connection is the series-parallel hybrid, and its passengers receive service individually. The most important thing is the distribution of service time. If the service time for the service station is $t$, the cumulative distribution function is $B(t)$, probability density function is $b(t)$. Generally includes fixed-length distribution $\mathrm{D}$, negative exponential distribution M, Erlang distribution ${ }_{E_{R}}$. Among them, the negative exponential distribution is commonly used. Since the service time does not depend on each other, it has the same negative exponential distribution after Kolmogorov-Smirnov test.

$$
b(t)= \begin{cases}\mu e^{-\mu t} & t \geq 0 \\ 0 & t<0\end{cases}
$$

In the above formula, $\mu>0$ and it is a constant.

\section{Composite Queuing Network Model}

According to the TSA, there is often one Pre-Check lane open for every three regular lanes in the current security systems, so we can divide the security system into several single units which consist of a pre-check lane and two regular check lanes. The flow chart of per unit can be seen in Figure 2. 


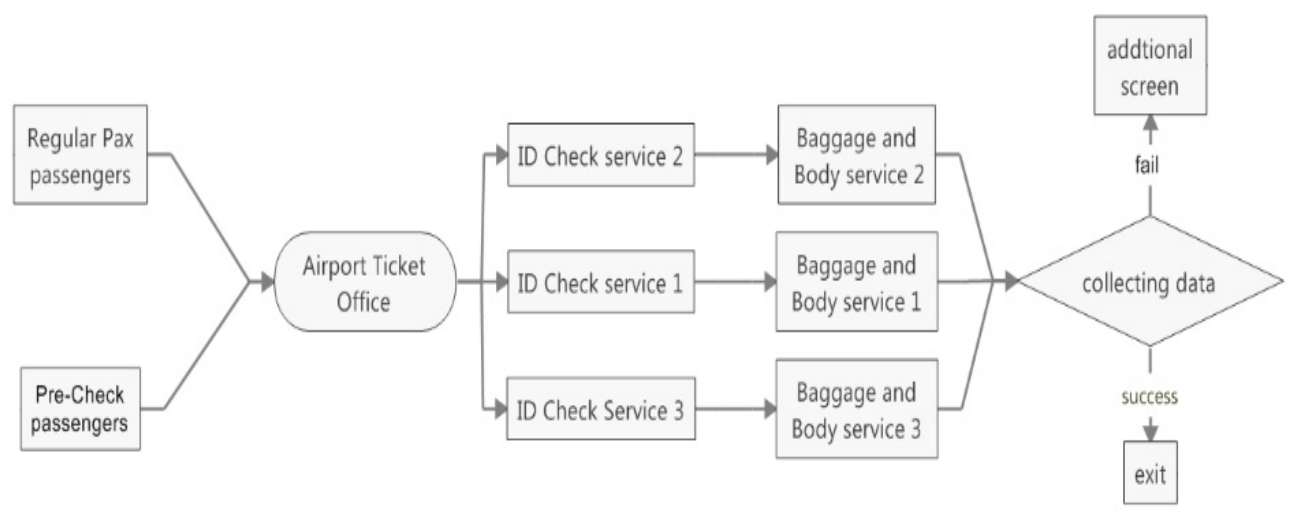

Figure 2. Simulation process of security system

In the whole unit, ID check service and baggage and body service are equivalent to a composite queuing system which consists of two queuing systems in series, and each unit is made up of three parallel composite queuing systems. Lane 1 and 2 are regular check lanes, while lane 3 is the pre-check lane. In this network, ID check process in regular check lanes can be seen as queuing model of $M / M / 2$, and the same process in pre-check lane Canberra seen as $M / M / 1$ model, and the equilibrium equations of system state probability are as follows.

$$
\left\{\begin{array}{l}
\sum_{n=0}^{\infty} P_{n}=1 \\
\mu P_{1}=\lambda_{i} P_{0} \\
(n+1) \mu P_{n+1}+\lambda_{i} P_{n-1}=\left(\lambda_{i}+n \mu\right) P_{n}(1 \leq n \leq c) \\
c \mu P_{n+1}+\lambda_{i} P_{n-1}=\left(\lambda_{i}+c \mu\right) P_{n}(n>c) \\
\rho=\frac{\lambda_{i}}{c \mu} \leq 1
\end{array}\right.
$$

Where $\lambda_{i}$ is the average number of arrived passengers per unit of time, and $i=1,2$, which represent regular lane and pre-check lane respectively, $\mu$ is the number of passengers served per unit of time, $c$ is the amount of service counters, $\rho$ is the degree of the system service strength.

According to the equilibrium equations, we can get that

$$
P_{0}=\left[\sum_{k=0}^{c-1} \frac{1}{k !}\left(\frac{\lambda_{i}}{\mu}\right)^{k}+\frac{1}{c !} \frac{1}{1-\rho}\left(\frac{\lambda_{i}}{\mu}\right)^{c}\right]^{-1}
$$

Where $k=n-c$.

The number of customers in the system is

$$
L_{s}=L_{q}+\frac{\lambda_{i}}{\mu}
$$

Where $L_{q}=\frac{(c \rho)^{c} \rho}{c !(1-\rho)^{2} \lambda_{i}} P_{0}$, which is the number of customers queuing for service in the system.

The average retention time spent in this system of passengers can be obtained, that is,

$$
w_{s}=\frac{L_{s}}{\lambda_{i}}=\frac{(c \rho)^{c} \rho}{c !(1-\rho)^{2} \lambda_{i}} P_{0}+\frac{1}{\mu}
$$

The average retention time spent in queuing per passenger is 


$$
w_{q}=\frac{(c \rho)^{c} \rho}{c !(1-\rho)^{2} \lambda_{i}} P_{0}
$$

After the ID check service, passengers will enter the baggage and body screening system, and continue to queue. Baggage and body screening system is made up of X-Ray scanning process and Millimeter Wave scanning process, and the structure chart of this system is shown in Figure 3.

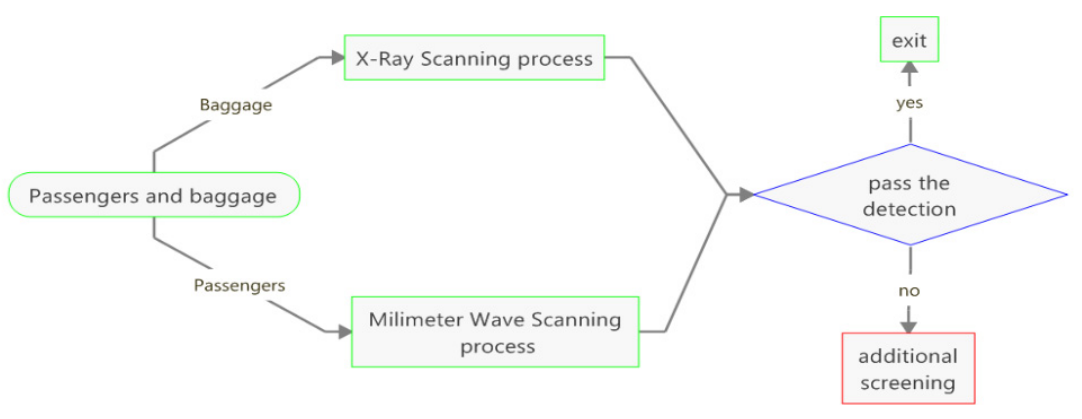

Figure 3. Structure of baggage and body screening process

In Figure 3, when passengers enter the baggage and body screening system, the baggage will be sent into X-Ray scanning process, while passengers will enter the Millimeter Wave scanning process. No matter which check failed, passengers should accept additional scanning with their baggage.

These two multipled process in Baggage and body screening system are both the same kind of queuing system with the single-service counter, which has the same arrival time intervals of passengers that are equal to the time for the customer to accept the service in ID check system, it means that they obey the same negative exponential distribution. The capacity of the system is $\mathrm{N}$, and the number of passengers served unit interval of X-Ray scanning process and Millimeter Wave scanning process in regular check lanes respectively are $\mu_{11}$ and $\mu_{12}$, while the same parameter in the pre-check lane are $\mu_{21}$ and $\mu_{22}$.

X-Ray scanning process is the $M / M / 1 / N / \infty$ queuing system, and its equilibrium equations of system state probability are as follows:

$$
\left\{\begin{array}{l}
\mu_{i 1} P_{1}=\mu P_{0} \\
\mu_{i 1} P_{n+1}+\mu P_{n-1}=\left(\mu+\mu_{i 1}\right) P_{n}(1 \leq n \leq N-1) \\
\mu_{i 1} P_{N}=\mu P_{N-1}
\end{array}\right.
$$

Where $i=1,2$. Considering that $\sum_{n=0}^{N} P_{n}=1$, we can get formulas of $P_{0}$ and $P_{n}$, that are,

$$
P_{0}=\left\{\begin{array}{ll}
\frac{1-\rho}{1-\rho^{N+1}} & \rho \neq 1 \\
\frac{1}{N+1} & \rho=1
\end{array} \text { and } P_{n}= \begin{cases}\frac{1-\rho}{1-\rho^{N+1}} \rho^{n} & \rho \neq 1 \\
\frac{1}{N+1} & \rho=1\end{cases}\right.
$$

Where $\rho=\frac{\mu}{\mu_{i 1}}$, which is the degree of the system service strength. The operating index of X-Ray Scanning process can be expressed by different functions that contain $P_{0}$ and $P_{n}$, that are, 


$$
\left\{\begin{array}{l}
L_{s 1}=\sum_{n=0}^{N} n P_{n}, \quad L_{q 1}=\sum_{n=1}^{N}(n-1) P_{n} \\
w_{s 1}=\frac{L_{s 1}}{\mu\left(1-P_{0}\right)}, w_{q 1}=w_{s 1}-\frac{1}{\mu_{i 1}}
\end{array}\right.
$$

Millimeter Wave Scanning process is a $M / M / 1 / N / \infty$ queuing system, the arrival time of its passengers equals to the passengers' of X-Ray Scanning process, so that formulas of $P_{0}$ and $P_{n}$ do not change. However, $\rho=\frac{\mu}{\mu_{i 2}}$ and the service time is different, which equal to $\mu_{12}$ and $\mu_{22}$ respectively. The operating indexes of Millimeter Wave Scanning process can be listed as follows:

$$
\left\{\begin{array}{l}
L_{s 2}=\sum_{n=0}^{N} n P_{n}, \quad L_{q 2}=\sum_{n=1}^{N}(n-1) P_{n} \\
w_{s 2}=\frac{L_{s 2}}{\mu\left(1-P_{0}\right)}, w_{q 2}=w_{s 2}-\frac{1}{\mu_{i 2}}
\end{array}\right.
$$

The retention time spent in baggage and body screening system is equal to the longest one between $w_{s 1}$ and $w_{s 2}$, that is,

$$
w_{s}^{*}=\max \left\{w_{s 1}, w_{s 2}\right\}
$$

\section{Numerical Simulation and Optimization}

\subsection{Simulation}

In this part, we use the data of TSA and preprocess the data. We apply the method of computer simulation to analyze the stability of the security system and identify bottlenecks. What's more, the composite queuing network model will be optimized.

By analyzing the data provided by TSA, we can get that $\lambda_{1}=0.0767, \lambda_{2}=0.109, \mu=0.07967$, $\mu_{11}=0.13295, \mu_{21}=0.2725, \mu_{12}=\mu_{22}=0.0895$, where $\lambda_{1}$ is the average number of arrived regular passengers per unit of time, and $\lambda_{2}$ is the average number of arrived pre-check passengers per unit of time. According to the above parameters, we build a simulation model by coding in MATLAB, and then we get the waiting time of all the three check lanes.

\subsubsection{Simulation Results of Regular Check Lane}

For the regular security lanes, the fluctuation of time that passengers spend in the ID check system can be seen in Figure 4, and the fluctuation of time that passengers spend in baggage and body screening system can be seen in Figure 5.

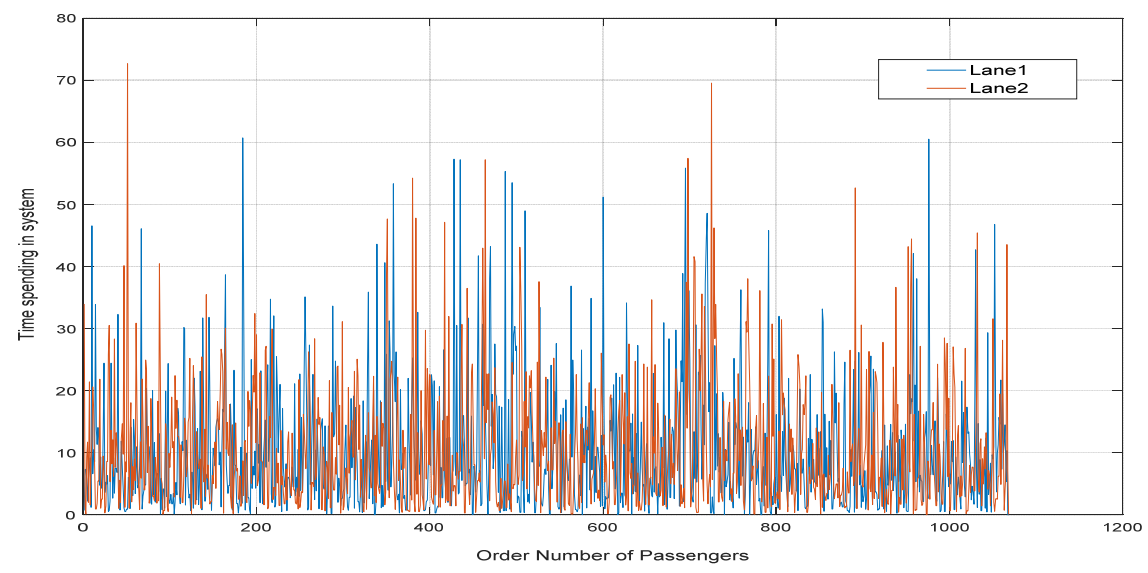

Figure 4. Time that regular pay spend in the ID check system 
From Figure 4, we can intuitively tell that the passenger's retention time in the system fluctuates around an average from this figure. And these two lanes do not have a big difference. What's more, the updating speed is normal, and the system is in a steady state.

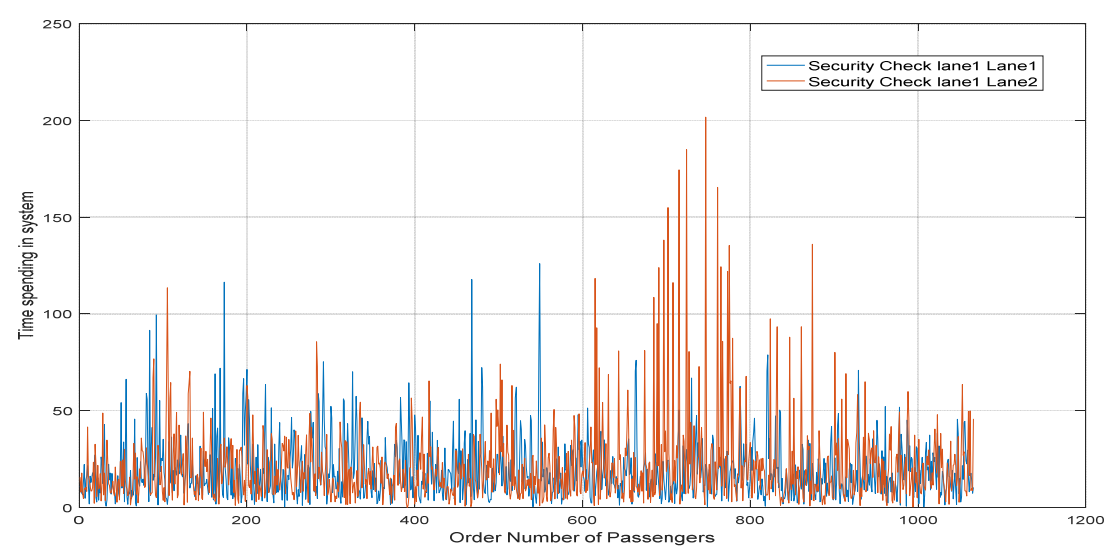

Figure 5. Time that regular pay spend in the Baggage and body screening system

It shows the changes of waiting time of different passengers in the Baggage and body screening system. Just like the Figure 4, the waiting time of different passengers varies. The total system is in a steady state with the queuing updating, and there is no malignant growth.

\subsubsection{Simulation Results Pre-Check Lane}

For the pre-check lane, the time that passengers spend in ID check system can be seen in Figure 6. And time that passengers spend in baggage and body screening system is shown in Figure 7.

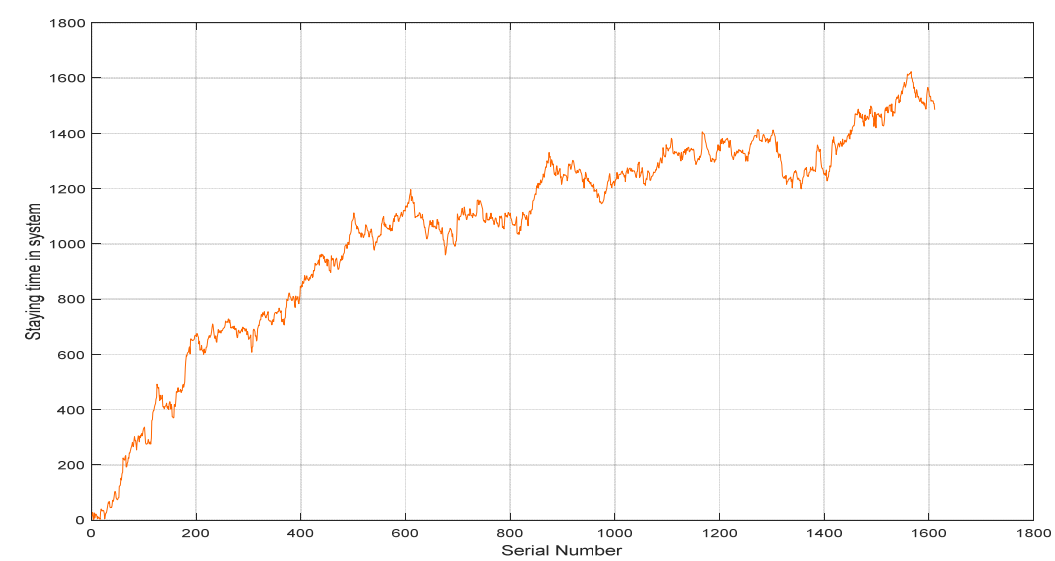

Figure 6. Time that TSA pre-check spend in the ID check system

It shows the distribution of the pre-checked passengers' retention time in the ID check system. The figure shows that the passengers' retention time and the length of queuing constantly increase as time goes by. The instability leads to the ID check system more and more crowded and eventually becomes the efficiency bottleneck of the airport security system. 


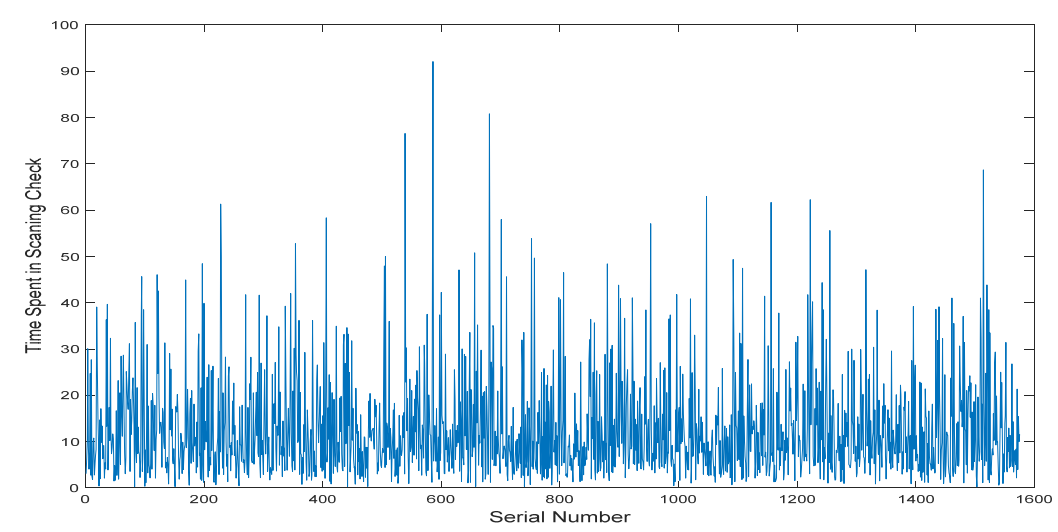

Figure 7. Time that TSA pre-check spend in the Baggage and body screening system

It shows the distribution of the pre-checked passengers' retention time in the Baggage and body screening system. Because the ID check system constraints plenty of passengers and there is no effect on the following system, the baggage and body screening system is in a steady state.

\subsection{System Optimization}

The bottleneck of the entire security system is the pre-check program. And the main constraint of the pre-check program is the ID check system. Therefore, we first carry out optimization analysis for the system.

\subsubsection{System Design Optimization}

Suppose the state is stable, the optimal service system is a $M / M / C$ system. The mean of the total cost of the system per unit time is as follows $(\mathrm{Hu}, 2012)$.

$$
z=c_{s} s+c_{w} L_{s}
$$

In the above formula, $z$ represents the total cost of the system per unit of time, $c_{s}$ represents cost per unit of

time for one service counter, $S$ represents the number of the service counter, $c_{w}$ represents the waiting cost for each passenger in the system per unit of time, $L_{s}$ represents the average length of queuing.

In the formula for the total cost, only the number of service stations is variable and other factors are determinate. So we can analyze the cost function $z=z(s)$ through marginal analysis method:

$$
\left.\begin{array}{l}
z\left(s^{*}\right) \leq z\left(s^{*}-1\right) \\
\mathrm{z}\left(s^{*}\right) \leq z\left(s^{*}+1\right)
\end{array}\right\}
$$

Combine the equation (6) and equation set (7), and we can obtain that:

$$
L\left(s^{*}\right)-L\left(s^{*}+1\right) \leq \frac{c_{s}}{c_{w}} \leq L\left(s^{*}-1\right)-L\left(s^{*}\right)
$$

Based on the above inequality, we acquire the optimal number of service counter in ID check system $s^{*}=2$ by the enumeration method realized in MATLAB.

According to the result of optimization, we can get Figure 8 and Figure 9 which can reflect the situation of passengers in the pre-check system after reform. 


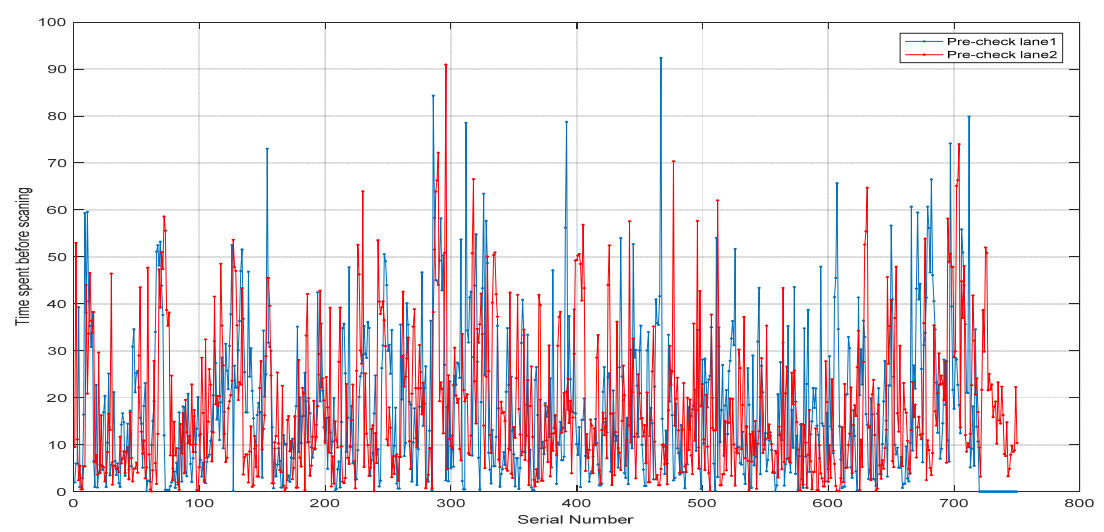

Figure 8. Time that TSA pre-check spend in the ID check system after reform

According to Figure 8, the passengers' waiting time fluctuates around a constant after the increasing of the ratio of pre-check lanes. It reflects that the problem of unstable caused by the pressure of ID check system has been solved. The ID check system of both pre-checked lanes and regular lanes are in a stable state.

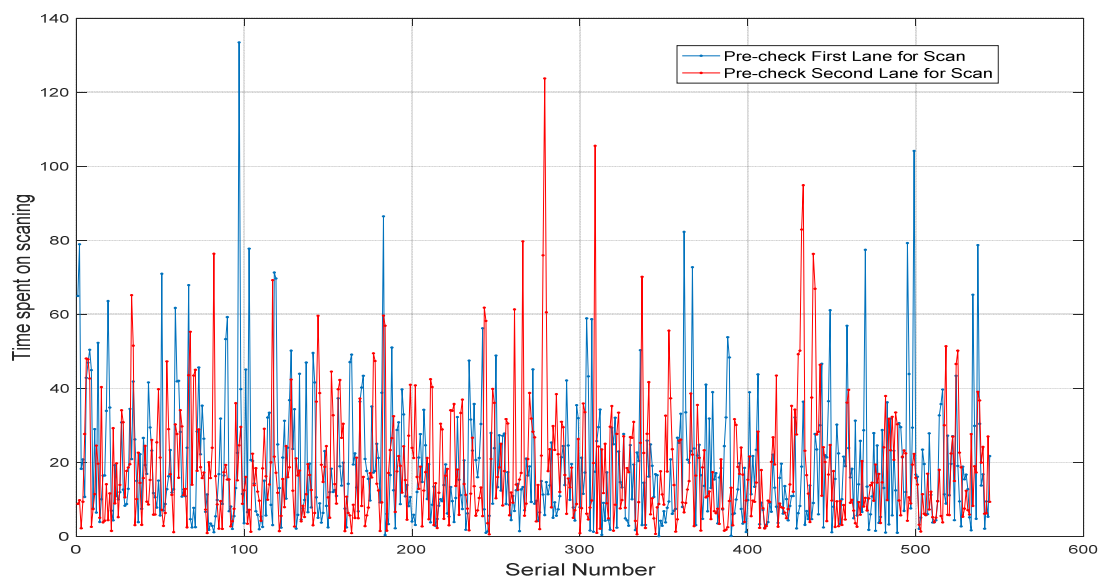

Figure 9. Time that TSA pre-check spend in Baggage and body screening system after reform

It reflects the fluctuation of simulated passengers' retention time of Baggage and body screening system in pre-check lanes after the optimization. The figure demonstrates that the system is in a stable state like regular security program. Comprehensive analysis of the simulation results shows that the security system is in a stable state and the stranded passengers are able to queue up for security. Besides, passenger throughput increased. So the reform measures for pre-check lanes are effective. However, considering the limitation of system capacity, we must reduce the variance of the retention time in this system, so we use the system control optimizing model to improve the service rate of millimeter scanning process.

\subsubsection{Optimization Model of Service Rate}

The distribution function of the longer service time is as $M=\max \left\{X_{1}, \ldots, X_{n}\right\}$ and $X_{1}, \ldots, X_{n}$ are independent of each other. The distribution function $M$ can be presented as:

$$
F_{\max }(\mathrm{z})=P\{M<z\}=P\{X \leq z, \mathrm{Y} \leq \mathrm{z}\}=F_{X}(\mathrm{z}) F_{Y}(\mathrm{z})
$$

After calculation, the distribution function of $M$ is as follows:

$$
F_{\text {max }}(\mathrm{z})= \begin{cases}\left(1-e^{-\alpha t}\right)\left(1-e^{-\beta t}\right) & t \geq 0 \\ 0 & t<0\end{cases}
$$


Therefore, the probability density function of $M$ is as follows:

$$
f_{\max }(t)=\frac{d}{d z} F_{\max }(\mathrm{z})= \begin{cases}\alpha e^{-\alpha t}\left(1-e^{-\beta t}\right)+\beta e^{-\beta t}\left(1-e^{-\alpha t}\right) & t \geq 0 \\ 0 & t<0\end{cases}
$$

So mean and variance of $M$ are

$$
\begin{gathered}
E(\mathrm{t})=\int_{0}^{+\infty}\left[\alpha e^{-\alpha t}\left(1-e^{-\beta t}\right)+\beta e^{-\beta t}\left(1-e^{-\alpha t}\right)\right] \\
\sigma^{2}=D(\mathrm{X})=E\left(\mathrm{X}^{2}\right)-[E(\mathrm{X})]^{2}
\end{gathered}
$$

According to the calculation formula of the length of queuing, we can obtain that (Wang, 2004):

$$
L_{q}=\sum_{n=1}^{N}(n-1) P_{n}
$$

The objective function is

$$
z=c_{s}^{*} \mu+c_{w} L_{s}
$$

In the above model, $L_{s}=L_{q}+\rho$ where $\rho=\frac{\lambda}{\mu}$ represents the service intensity.

According to the references, we obtain the service cost for one passenger per unit of time $c_{s}=5.6$, the cost of each passenger for staying per unit of time in this system $c_{w}=6.818$. Based on the above calculation process, we consider using the particle swarm optimization to optimize the objective function in MATLAB. Figure 10 shows the shape of the function of the total cost which is optimized by the PSO.

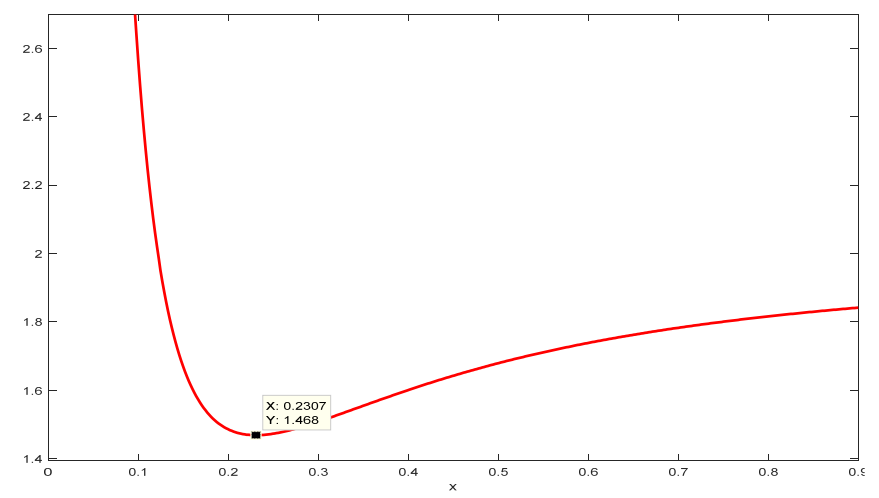

Figure 10. Total cost function graph of Baggage and body screening system

The optimal solution of the objective function is obtained at the cursor point in the figure. Under this circumstances, the service rate of Millimeter wave scan process is $\mu_{1}^{*}=0.2307$, the minimum total cost per unit time is $z_{\min }=1.4682$. Besides, the optimal service rate of baggage and body screening system is $\mu^{*}=0.1662$. And the simulating result is shown in Figure 11. 


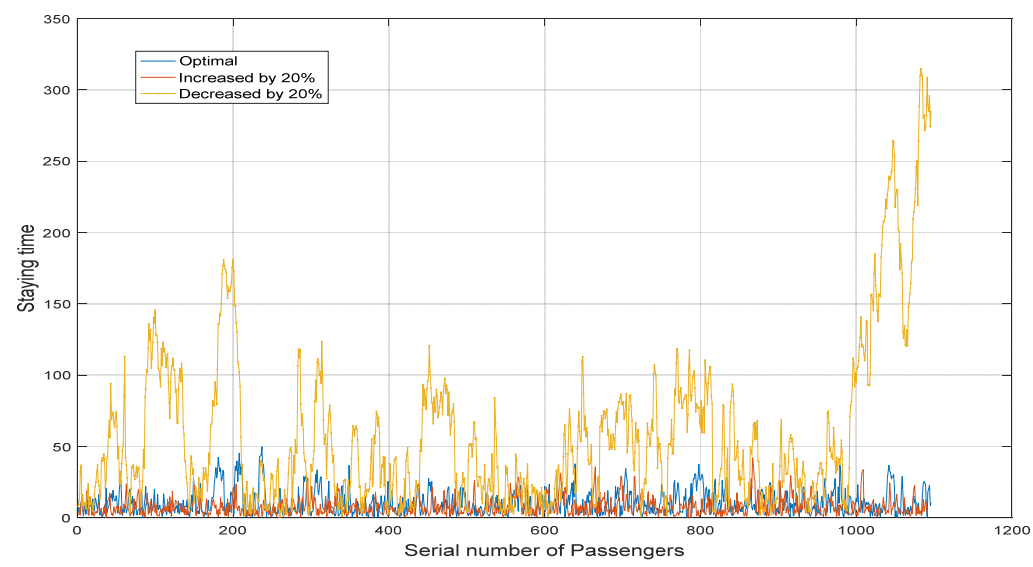

Figure 11. The simulation results of the baggage and body screening system under different service rates

From Figure 11, we can see that the variance of retention time in baggage and body scanning system is reduced, and the system is stable. In practice, we only need to improve the service rate as much as possible towards to the direction of the optimal value. For further study, we should consider the effect of culture and region which makes a difference to the behavior of local people.

\section{Conclusion and Suggestion}

In this paper, we establish a composite queuing network model based on the queuing theory and analyze the stability of the security system combining the real situation of at Chicago's O' Hare international airport. In considering the different security program that exists in the airport, we divide the security system into many units which consist of two regular check lanes and one pre-check lane based on the proportion of different lanes. We depict the inter-relationship of the ID check process, X-ray scanning process and Millimeter wave scanning process in combination of the reality and establish a composite queuing network model. Then we apply the model into the numerical simulation and analyze the fluctuation of passengers' retention times. We find that these two regular check lanes are in a stable state and the pre-check lane is instability. In pre-check lane, the retention time of passenger rapid growth which leads to the length of queue constantly increase so that passengers cannot pass the security check-in time.

In order to eliminate bottlenecks, we using the system design optimization model in the process of ID check in the pre-check lane, optimize the amount of ID check service counters under the target of keeping the system stability with the lowest costs. According to the result of optimization, we change the proportion of pre-check lanes in the security system and compare the results of the simulation with original results to verify the effectiveness of our model. By analyzing the difference of the results, we draw the conclusion that the proportion of pre-check lanes should be improved. Finally, we reduce the variance of retention time in the baggage and body screening system using the system control optimizing model and give out the result of simulating.

\section{Acknowledgments}

While remaining responsible for any errors in this paper, the authors would like to thank guidance and advice of Dr. Yuanbiao Zhang on preparing the article.

\section{Reference}

Araujo, G. E., \& Repolho, H. M. (2015). Optimizing the Airport Check-In Counter Allocation Problem. Journal of Transport Literature, 9(4), 15-19. https://doi.org/10.1590/2238-1031.jtl.v9n4a3

Cheng S. W., Zhang, Y. P., \& Bie, Y. M. (2015). Dynamic allocation of resources and dispatching intelligent theory of civil airport terminal. Electronic Industry Press.

Chun, H. W., \& Mak, R. W. T. (1999). Intelligent resource simulation for an airport check-in counter allocation system. IEEE Transactions on Systems, Man, and Cybernetics, Part C (Applications and Reviews), 29(3), 325-335. https://doi.org/10.1109/5326.777069

Gkritza, K., Niemeier, D., \& Mannering, F. (2006). Airport security screening and changing passenger satisfaction: An exploratory assessment. Journal of Air Transport Management, 12(5), 213-219. https://doi.org/10.1016/j.jairtraman.2006.03.001 
Gu, G. H., Xu, L., \& Xia, Q. W. (2011). Research on flight simulation of terminal departure. Science \& Technology Information, 33, 91-92.

Guo, Y. Y. (2013). Forecast of outbound passenger flow of terminal based on chaotic time series (Doctoral dissertation). Harbin: Harbin Institute of Technology.

Han, Z. G. (2005). Mathematical modeling method and its application. Higher Education Press.

Hon, W. C., \& Mak, R. W. T. (1999). Intelligent resource simulation for an airport check-in counter allocation system. IEEE Transactions on Systems, Man and Cybernetics, Part C (Applications and Reviews), 29(3), 325-335. https://doi.org/10.1109/5326.777069

$\mathrm{Hu}$, Y. Q. (2012). Operations research tutorial. Tsinghua University Press.

Hwang, T. L., Jeng, C. R., \& Wang, S. S. (2012). Airport check-in counter assignment: A proposed solution. International Journal of Aviation Management, 1(4), 257. https://doi.org/10.1504/IJAM.2012.050473

Kiylld, R. K., \& Karasahin, M. (2008). The capacity analysis of the check-in unit of Antalya Airport using the fuzzy logic method. Transportation Research Part A: Policy and Practice, 42(4), 610-619. https://doi.org/10.1016/j.tra.2008.01.004

Li, N. Y. (2012). Research on dynamic simulation of terminal departure flight. Technology Wind, 12, 40-41.

Pašagić, Š. J., Drljača, M., \& Kavran, Z. (2013). Logistics of International Express Shipping and Air Traffic. PROMET-Traffic \& Transportation, 25(3).

Regattieri, A., Gamberini, R., Lolli, F., \& Manzini, R. (2009). Designing production and service systems using queuing theory: Principles and application to an airport passenger security screening system. International Journal of Services and Operations Management, 6(2), 206-225. https://doi.org/10.1504/IJSOM.2010.030636

Široký, J., \& Hlavsová, P. (2014). Optimizing process of check-in and security check at airport terminals. Department of Transport Technology and Control, 9(4), 118-128.

Takakuwa, S., \& Oyama, T. (2003, December). Modeling people flow: Simulation analysis of international-departure passenger flows in an airport terminal. In Proceedings of the 35th conference on winter simulation: Driving innovation (pp. 1627-1634). Winter Simulation Conference.

Van Dijk, N. M., \& Van Der Sluis, E. (2006). Check-in computation and optimization by simulation and IP in combination. European Journal of Operational Research, 171(3), 1152-1168. https://doi.org/10.1016/j.ejor.2005.01.023

Wang, B. T. (2004). Fundamentals of Mathematical Modeling. Beijing Jiaotong University press.

Yan, S., Tang, C. H., \& Chen, M. (2004). A model and a solution algorithm for airport common use check-in counter assignments. Transportation Research Part A: Policy and Practice, 38(2), 101-125. https://doi.org/10.1016/j.tra.2003.10.001

Yang, Y. H., \& Gao, J. H. (2005). Application of queuing theory in capacity analysis of terminal. Journal of Civil Aviation University of China, z1, 89-90.

\section{Copyrights}

Copyright for this article is retained by the author(s), with first publication rights granted to the journal.

This is an open-access article distributed under the terms and conditions of the Creative Commons Attribution license (http://creativecommons.org/licenses/by/4.0/). 